\title{
Vibronic coupling model to calculate the photoelectron spectrum of phenol
}

\author{
M. P. Taylor \\ School of Chemistry, University of Birmingham, Birmingham, B15 2TT, U.K. \\ G. A. Worth* \\ Dept. of Chemistry, University College London, 20 Gordon Street, London \\ WC1H OAJ, U.K.
}

\begin{abstract}
The photoelectron spectrum for the two lowest ionisation bands of phenol has been simulated using quantum dynamic methods. A vibronic coupling Hamiltonian was set up consisting of seven vibrational modes and the first two ionised states. Parameters for the model are obtained by fitting adiabatic surfaces to a series of points calculated using ab initio methods. Such a model allows non-adiabatic couplings between the states to be included. CASSCF calculations used in this work provide reliable quantum chemical information for the model and the calculated photoelectron spectrum shows good agreement to experiment. The vibrational fine structure of both bands are reassessed and different assignments to those previously reported are detailed. The existence of a conical intersection between the ionised states is reported and its role in the dynamics of phenol upon ionisation is examined.
\end{abstract}

Key words: phenol photochemistry, wavepacket dynamics, conical intersection, photoelectron spectrum

\section{Introduction}

Like benzene, phenol and its derivatives are abundant in compounds found naturally and synthesised industrially. As a result, phenol has been extensively studied through theoretical and experimental means. In recent decades, much attention has been given to the photochemistry and photodissociation of phenol [1-6]. The dynamics along the $\mathrm{O}-\mathrm{H}$ stretching mode involving different electronic states provide examples of non-adiabaticity and conical intersections. The two lowest lying excited states $\left({ }^{1} \pi \pi^{*}\right.$ and $\left.{ }^{1} \pi \sigma^{*}\right)$ have been shown 
to contain a conical intersection seam $[1,7]$. This enables ultrafast internal conversion from the bright ${ }^{1} \pi \pi^{*}$ state to the ${ }^{1} \pi \sigma^{*}$ state, which leads to the dissociation of hydrogen to form the phenoxyl radical. There is also evidence to suggest the $\mathrm{O}-\mathrm{H}$ dissociation bypasses the conical intersection through hydrogen tunnelling $[3,8]$. There also exists another conical intersection between the ${ }^{1} \pi \sigma^{*}$ and $\mathrm{S}_{0}$ state, which provides an alternative pathway of rapid relaxation to the electronic ground state.

Following the seminal work of Sobolewski and Domcke [9], the existence of dissociative ${ }^{1} \pi \sigma^{*}$ states has been shown for a range of aromatic molecules. They are dark states, characterised at the Franck-Condon (FC) point as a 3s Rydberg state, that forms a conical intersection with the ground-state. Moreover, their repulsive nature is responsible for hydrogen transfer or dissociation in many bioaromatic molecules [10] and is thought to provide photostability. Whilst this state cannot be directly accessed, its position relative to the ${ }^{1} \pi \pi^{*}$ state dictates the dynamics that occurs and the location of the crossing points. This has been observed in a variety of planar organic molecules $[9,11]$. This difference in positions of the two states and the effect becomes apparent when comparing the photodissociation of phenol with pyrrole $[10,12,13]$. The shift in relative energy of the ${ }^{1} \pi \sigma^{*}$ with respect to the ${ }^{1} \pi \pi^{*}$ state in phenol has also been investigated when complexed with water and ammonia clusters [14]. While the photo-excited dynamics of neutral phenol has been extensively studied, the photo-ionisation and subsequent dynamics has received little attention and the ion states are usually only included as a continuum for the theoretical description of pump-probe measurements on the excited states $[15,16]$. Recent work has also recorded the effect of aqueous solvation on the ionisation and excited states of phenol [17]. The photoelectron spectrum has been measured and the ionisation potentials determined to be 8.5 and $9.28 \mathrm{eV}$ for the first two ion states [18-21]. The symmetry of the ion states have been determined to be ${ }^{2} B_{1}$ and ${ }^{2} A_{2}$ and result from the removal of a $\pi$ electron. The full $\mathrm{He}(\mathrm{I}) / \mathrm{He}(\mathrm{II})$ spectra were measured with the aim of obtaining all the valence ionisation potentials and the vibrational fine structure of the photoelectron spectrum has been largely overlooked. The possibility of non-adiabaticity and coupling associated with the ionised states has also not been explored.

Quantum dynamics simulations, which solve the time-dependent Schrödinger equation, are a powerful way to probe the photodynamics of a system and provide rationale behind experimental observables [22]. Examples are the use of simulated spectra and population dynamics to support pump-probe timeresolved photoelectron spectra (TR-PES) $[23,24]$. These calculations require a potential energy function in the form of a set of coupled surfaces to model the excited-state dynamics. In some studies the time-resolved signal from a TR-PES experiment has been directly modelled [25-27] and in this case the potential surfaces of the ion are also required. 
Coupled potential surfaces are impossible to obtain accurately for more than a few atoms due to large number of high level quantum chemistry calculations required and the difficulty of fitting these points using multi-dimensional functions. For this reason, model Hamiltonians are a useful approach to capture the essential physics in a simple way. For the coupled surfaces involved in photoexcited systems the vibronic coupling model Hamiltonian $[28,29]$ has become a standard approach. This involves a low-order Taylor expansion of diabatic potentials and couplings with parameters obtained by fitting to quantum chemistry calculations and experimental data. Making use of the vibronic coupling Hamiltonian thus allows for explicit consideration of vibronic couplings and non-adiabatic photochemistry. We have already applied the vibronic coupling Hamiltonian to a range of aromatic systems [30-34].

In this paper, a simple vibronic linear coupling model is constructed to simulate the photoelectron spectrum of the two lowest ionisation bands of phenol. This model allows the dynamics to be elucidated in terms of normal modes and couplings between the diabatic electronic states. It was found that a modest, seven mode model was sufficient to replicate the necessary photodynamic behaviour. The vibrational structure of the obtained photoelectron spectrum has been assigned and compared with previous studies. Differences between the assignments have been noted and discussed. The possibility of a conical intersection between the states is investigated, and its affect on the dynamics can be observed by following the relative diabatic state populations. These surfaces can be used, together with potential surfaces for phenol $[1,6]$ to help support the TR-PES of phenol measured in recent work by Riley et al. [17] which requires ionisation to two closely lying ion states.

\section{Methodology}

\subsection{Computer Simulation Details}

\subsubsection{Model Hamiltonian}

By making use of the vibronic coupling Hamiltonian [28], the diabatic potentials can be expressed, through a Taylor series, in terms of dimensionless normal modes around a particular point, $Q_{0}$, usually taken as the equilibrium geometry. At this point, the diabatic electronic wavefunction is assumed to be equal to the adiabatic. The Hamiltonian can therefore be written

$$
\mathbf{H}=\mathbf{H}^{(0)}+\mathbf{W}^{(0)}+\mathbf{W}^{(1)}+\ldots
$$


with the zeroth-order Hamiltonian expressed as the ground state Hamiltonian in the Harmonic approximation

$$
H^{(0)}=\sum_{\alpha} \frac{\omega_{\alpha}}{2}\left(\frac{\partial^{2}}{\partial Q_{\alpha}^{2}}+Q_{\alpha}^{2}\right)
$$

and $\omega$ representing the frequency of mode $Q$. The set of diabatic coupling matrices, $W$ describe the changes in excited state surfaces compared with the ground state. $W^{(0)}$ is the ground state Harmonic oscillator displaced to excitation energy, $E_{i}$. The first order matrix elements are expressed as

$$
\begin{gathered}
W_{i i}^{(1)}=\sum_{\alpha} \kappa_{\alpha}^{(i)} Q_{\alpha} \\
W_{i j}^{(1)}=\sum_{\alpha} \lambda_{\alpha}^{(i, j)} Q_{\alpha} \quad ; \quad i \neq j
\end{gathered}
$$

Here, the $\kappa$ parameters are related to the gradients of the adiabatic potential at $Q_{0}$ with respect to nuclear coordinates and the $\lambda$ parameters are the nonadiabatic elements, again at $Q_{0}$, which provide coupling between the electronic states. Included also were the on-diagonal second order matrix elements,

$$
W_{i i}^{(2)}=\sum_{\alpha} \frac{1}{2} \gamma_{\alpha}^{(i)} Q_{\alpha}^{2}+\sum_{\alpha<\beta} \gamma_{\alpha \beta}^{(i)} Q_{\alpha} Q_{\beta}
$$

where $\gamma$ are the second derivatives of the adiabatic potentials at $Q_{0}$. These parameters provide changes in the frequencies and Duschinsky rotation by coupling the modes.

For modes exhibiting strong anharmonicity it becomes necessary to express the diabatic potentials as Morse potentials in place of the harmonic form above. These have the following form

$$
V=D_{0}\left[\exp \left(\alpha\left(Q-Q_{0}\right)-1\right)\right]^{2}+E_{0}
$$

with parameters $D_{0}$ for the dissociation energy, $Q_{0}$ for the equilibrium geometry, and $\mathrm{E}_{0}$ an energy shift.

Consideration of molecular symmetry is important as many of the matrix elements vanish due to symmetry arguments

$$
\begin{gathered}
\kappa_{\alpha}^{(i)} \neq 0 \quad \text { if } \Gamma_{\alpha} \supset \Gamma_{A} \\
\lambda_{\alpha}^{(i, j)} \neq 0, \quad \text { if } \quad \Gamma_{\alpha} \otimes \Gamma_{i} \otimes \Gamma_{j} \supset \Gamma_{A}
\end{gathered}
$$




$$
\gamma_{\alpha \beta}^{(i)} \neq 0, \quad \text { if } \quad \Gamma_{\alpha} \otimes \Gamma_{\beta} \otimes \Gamma_{i} \supset \Gamma_{A}
$$

where $\Gamma_{\alpha}, \Gamma_{\beta}$ are the symmetry irreps of the vibrations, $\Gamma_{i}, \Gamma_{j}$ the irreps of the electronic states and $\Gamma_{A}$ the totally symmetric irrep. Phenol possesses $\mathrm{C}_{\mathrm{s}}$ symmetry, however for determining the modes that couple the ${ }^{2} B_{1}$ and ${ }^{2} A_{2}$ ionised states and give rise to vibrational fine structure, the symmetry was ascended to $\mathrm{C}_{2 \mathrm{v}}$. From the symmetry arguments only the totally symmetric $a_{1}$ modes and the $b_{2}$ coupling modes need to be considered.

The potential energy surfaces and parameters are obtained through a leastsquares fitting procedure to a series of ab initio points calculated along each normal mode. This is done using the VCHAM program [35] within the Quantics package [36]. Energies at each of these points were calculated at the CASSCF level of theory, employing an $(7,8)$ active space with a $6-31+\mathrm{G}^{*}$ basis. The active space comprises the oxygen lone pair, $\pi \pi, \pi \pi^{*}$ and $\pi \sigma^{*}$ orbitals. All electronic structure calculations were run using the Gaussian 09 program [37].

\subsubsection{Calculating the spectrum}

The photoelectron spectrum is obtained by taking the Fourier transform

$$
I(\omega) \propto \omega \int_{-\infty}^{\infty} \mathrm{d} t C(t) \mathrm{e}^{i \omega t}
$$

of the autocorrelation function

$$
\begin{aligned}
C(t) & =\langle\Psi(0) \mid \Psi(t)\rangle \\
& =\left\langle\Psi(t / 2)^{*} \mid \Psi(t / 2)\right\rangle
\end{aligned}
$$

To reduce spurious structures (Gibbs phenomenon) that result from the finite propagation time in the Fourier transform, the autocorrelation function is multiplied by a weight function $\cos (\pi t / 2 T)$. The broadening seen in experimental spectra can then be simulated by further multiplying with a damping function $\exp (-t / \tau)$.

The quantum dynamics were performed with the Quantics package which uses the MCTDH method, a highly efficient algorithm for the propagation of wavepackets [38]. The wavepacket propagations were initiated from separate excitations to the ${ }^{2} B_{1}$ and ${ }^{2} A_{2}$ states and were performed using seven modes. Initially each wavepacket was prepared as a Gaussian with a defined width and centred on the Franck-Condon point. Each propagation was performed for 200 fs. Each mode in the simulation was described using a Harmonic oscillator 
discrete variable representation (DVR) [38]. Details of the basis sets used are given in Table 1.

\section{Results}

\subsection{Fitted Parameters}

The calculated vertical ionisation energies are shown in Table 2. Compared with experimental data, the energies obtained are underestimated but have a good agreement in the relative energetic difference between the ${ }^{2} B_{1}$ and ${ }^{2} A_{2}$ states. For use in the model Hamiltonian, the energies were shifted to match those experimentally measured $[21,39]$.

The vibrational frequencies, evaluated at the MP2/aug-cc-pVDZ level of theory are listed in Table 3 . The assignment of these frequencies to $C_{2 v}$ symmetry followed those outlined by Evans [40] and correspond well to other reported values [41-44]. We do note however that in this work phenol has been defined along a plane such that the $b_{2}$ modes are in plane vibrations and the $b_{1}$ modes are out of plane. Vibrational modes with significant motion of the phenyl ring and $\mathrm{C}-\mathrm{O}$ moiety are described as $\mathrm{X}$-sensitive modes and are greatly affected by the substituent $\mathrm{X}[40,45]$, which in this case is oxygen. These modes are $Q_{1}, Q_{2}, Q_{7}, Q_{13}, Q_{27}$ and $Q_{29}$.

A quantum dynamics model containing all 33 normal modes of phenol would be prohibitively demanding and so only those important in describing the photochemistry of phenol were included. From symmetry arguments the $a_{1}$ and $b_{2}$ modes are likely to contribute most to the dynamics. From these there are seven modes important to the phenol model. They are the low frequency $b_{2}$ in plane $\mathrm{C}-\mathrm{O}-\mathrm{H}$ bend and ring deformation modes, the lowest frequency $a_{1}$ ring deformation/C-O stretch mode and the $a_{1}$ modes corresponding to the $\mathrm{O}-\mathrm{H}$ bend, $\mathrm{C}-\mathrm{O}$ and $\mathrm{C}-\mathrm{C}$ stretch, $\mathrm{C}-\mathrm{C}$ ring stretch and the $\mathrm{O}-\mathrm{H}$ stretch. These are illustrated in Figure 1. Previous models to study the excited states of phenol consider two nuclear degrees of freedom $\mathrm{r}$ and $\theta$ corresponding to the $\mathrm{O}-\mathrm{H}$ bond length and $\mathrm{O}-\mathrm{H}$ torsion $[1,4,46]$. These degrees of freedom are present in the model described here as vibrational normal modes.

The parameters obtained from fitting the vibronic coupling model Hamiltonian to the adiabatic surfaces are shown in Table 4 and in the supplementary information. Cuts along the seven important normal modes are shown in Figure 2. The quality of the fitting is evident by how well the curves pass through the calculated points, particularly around points of interest such as the minimum or crossing points. Quantitatively the quality of fitting can be assessed 
by the root mean square displacement (RMSD) between the fitted surfaces and the $a b$ initio points. For the fitted surfaces in the model Hamiltonian the RMSD was $0.022 \mathrm{eV}$.

The on-diagonal linear coupling constants, $\kappa_{\alpha}$, are shown in Table 4 . For the totally symmetric $a_{1}$ modes the values are all non-zero. The three highest frequency modes were subsequently fitted as Morse potentials to account for anharmonicity. Details of these parameters can be found in the supplementary information. Consideration of the parameter ratio $\kappa_{\alpha} / \omega_{\alpha}$ is important for determining how displaced the ionised state minima are from the ground state minimum. This parameter becomes important for lower frequency modes thus is largest for $\nu_{1}$ with important contributions coming from $\nu_{6}$ and $\nu_{7}$. The C-C ring stretch mode, $\nu_{9}$ was also chosen as being important. Despite having a lower parameter ratio, the size of the displacement from the equilibrium geometry was significant. An adjustment to the value of this mode improved the simulated spectrum and also increased the parameter ratio. The new value is listed in Table 5.

Table 4 lists the off-diagonal linear coupling constants, $\lambda_{\alpha}$, the coupling between electronic states. Based on the symmetries of the two ionised states, non-zero values are expected for the $b_{2}$ modes. Making use of the ratio $\lambda_{\alpha} / \omega_{\alpha}$ again indicates low frequency modes to be of most importance. The only modes to exhibit significant coupling are the two lowest frequency $b_{2}$ modes. On inspection, it was found the initial values for these modes to be insufficient to reproduce the experimental spectrum. The values listed have been adjusted (Table 5) such that good agreement with experiment is achieved.

The on-diagonal second order quadratic parameters, $\gamma_{\alpha \alpha}$, and the bi-linear parameters, $\gamma_{\alpha \beta}$, are listed in the supplementary information. The former parameters correspond to changes in frequency from the ground state to the ionised states, whilst the latter are responsible for intramolecular vibrational relaxation (IVR). These values are typically small although several of the bilinear parameters exhibited large values. It was necessary to adjust some of these parameters to obtain spectra with good experimental agreement.

A Morse potential was required to fit several of the high frequency $a_{1}$ modes, parameters of which can be found in the supplementary information. These modes were the $\mathrm{C}-\mathrm{H}$ stretches $\nu_{10}$ and $\nu_{11}$ and the $\mathrm{O}-\mathrm{H}$ stretch, $\nu_{12}$. The linear coupling constants (gradient at the Franck-Condon point) obtained from the fitting are again useful indicators of the importance of these modes. All the values are very small except for the upper ionised state of $\nu_{12}$ and only this mode, the $\mathrm{O}-\mathrm{H}$ stretch, was included in the model. Based on these arguments, the remaining modes were neglected from the model Hamiltonian as they exhibit little coupling and would not be expected to contribute to the spectrum. 


\subsection{Cuts through the potential energy surface}

Figure 2(a) shows the cut along the ring deformation/C-O stretch $\nu_{1}$ mode. As expected for a totally symmetric mode, the on-diagonal linear coupling value is non-zero and a large shift is observed in the ${ }^{2} B_{1}$ state. Another significant feature seen along this mode is the crossing of the states suggesting a conical intersection to be present. The existence and location of such a crossing is discussed later. The two $b_{2}$ coupling modes are shown in Figure 2(b,c). Being non totally symmetric modes there is no shift away from $Q_{0}$, however a small change in frequency is noted for both modes.

The totally symmetric O-H bend mode (Figure 2(d)) and C-O stretch (Figure $2(\mathrm{e})$ ) show little significance aside from the expected shift away from the equilibrium geometry. Both cuts, particularly mode $\nu_{7}$ contain calculated points in the ${ }^{2} A_{2}$ state that lie away from the fitted curve. Calculated points along the next ionised state energetically present no crossing or interaction between states and we attribute the awry points as minor failings of the low level CASSCF calculations. As these points are located far from the equilibrium geometry, they are expected to have little effect.

Of particular interest was the cut along the $\mathrm{C}-\mathrm{C}$ ring stretch mode, $\nu_{9}$, Figure 2(f). A significant shift in equilibrium geometry is seen for the ${ }^{2} B_{1}$ state whilst a smaller shift in the opposite direction for the ${ }^{2} A_{2}$ causes the two states to become close. As expected, the cut along the O-H stretch (Figure 2(g)) is anharmonic for each state and best fitted as a Morse potential. Unlike in the $S_{1}, S_{2}$ and $S_{3}$ excited states of neutral phenol, the ionised states show no evidence of crossing or photodissociation of the hydrogen.

It is evident that for the totally symmetric modes large shifts are present only for the lower ${ }^{1} B_{2}$ states and this is reflected in the values of on-diagonal linear coupling parameters. The consequence of this is likely to appear in a spectrum containing significant vibrational structure from several progressions whilst the ${ }^{2} A_{2}$ spectrum may lack structure.

The photoelectron spectrum calculated using the parameters obtained directly from the fitting is shown in Figure 3. When compared with experiment (reproduced in Figure 6(a)), it is clear the simulation does not accurately reproduce the spectrum. The likely causes for this are inadequacies in the fitted values and therefore require adjustments. Table 5 lists the parameters where an adjustment from the fitted values was necessary in order to successfully reproduce the experimental spectra.

Examination of the ${ }^{2} B_{1}\left(D_{0}\right)$ band shows the simulated spectrum to be dominated by a progression of a single mode, $\nu_{1}$ only. In contrast, the experimental spectrum shows there to be several different progressions present. The general 
shape and range of this band in the simulated spectrum matches well with experiment but the correct fine structrue is lacking. This indicates adjustments are needed to the on-diagonal linear coupling constants of the other totally-symmetric modes to incorporate the fine structure. A change to the on-diagonal linear coupling constant for $\nu_{9}$ for the ${ }^{2} B_{1}$ state ensured the first peak in this progression conformed correctly to being the most intense peak. This change, whilst retaining a similar value to the fitted value, is particularly notable as a change in sign was required.

In altering the sign of $\kappa_{9}^{(1)}$, the implication is a change in the direction of the gradient and a large shift in the minimum along this mode. This alludes to a failing in the CASSCF potential energy surfaces. It is, however, a necessary change as simulations performed with a positive value of $0.2 \mathrm{eV}$ produce a spectrum similar to Figure 3, devoid of any additional fine structure except a single progression from $\nu_{1}$. Support for this change is given by calculations at the MP2/6-31+ $\mathrm{G}^{*}$ level of theory. Here, the gradient along $\nu_{9}$ for the ${ }^{2} B_{1}\left(D_{0}\right)$ state at the neutral equilibrium geometry calculated gives a value of $\kappa_{9}^{(1)}=$ $-0.214 \mathrm{eV}$, close to the altered value for this parameter. This points to the potential importance of electron correlation for this mode.

The change in this parameter also serves to change the $\nu_{1}$ progression, preventing it from dominating the fine structure and moving the position of the most intense peak. As such, the emergence of other peaks, from different vibrational progressions, without changing any other on-diagonal linear coupling constants supports the need to change the $\nu_{9}$ parameter in such a way.

The ${ }^{2} A_{2}\left(D_{1}\right)$ band simulated using the unaltered parameters is vastly different to experiment. Instead of a featureless spectrum comprising two peaks, the simulation incorrectly produces addition peaks of differing intensities. It was found that no adjustments to the $\kappa$ values were required to reproduce experiment, but rather alterations to the off-diagonal linear, $\lambda_{\alpha}$ and bi-linear, $\gamma_{\alpha \beta}$ coupling constants.

The changes to the off-diagonal bi-linear parameters prevented the spurious progressions from occurring and ensured the relative peak heights were in good agreement. The presence of large values in these parameters would often result in too much broadening to the ${ }^{2} A_{2}$ band and loss of the little structure present. Furthermore, these adjustments make certain the origin is the most intense peak in the band and occurs at the correct energy. These changes, despite being successful in reproducing the correct structure, however, often resulted in spectra that are too structured and lacked broadness. As discussed above, an increase in the coupling between the electronic states from the low frequency $b_{2}$ modes was needed to broaden the spectrum. This increase in coupling had no effect on the the ${ }^{2} B_{1}\left(D_{0}\right)$ band but was required for the ${ }^{2} A_{2}\left(D_{1}\right)$ band, although the effect on the spectrum is small, but important. 
Since the strength of the coupling corresponds to the square of the parameter, then increasing the parameter by a magnitude is needed in order to increase the coupling sufficiently.

\subsection{Conical Intersection}

To check whether a conical intersection exists between the ${ }^{2} B_{1}$ and ${ }^{2} A_{2}$ states a CAS $(7,8)$ conical intersection minimisation was performed using Gaussian09. The point in normal mode space corresponding to the intersection is denoted $Q_{\mathrm{CI}}$. As a function of the normal modes coordinates, the conical intersection energy minimum can be expressed as: $Q_{1}=-1.31, Q_{2}=0.88, Q_{3}=0.15$, $Q_{4}=-0.14, Q_{5}=-0.59, Q_{6}=1.52, Q_{7}=0.51, Q_{9}=0.96, Q_{10}=0.14$, $Q_{11}=0.40, Q_{12}=-1.86, Q_{13}=1.33, Q_{14}=0.61, Q_{17}=-0.54, Q_{18}=0.58$, $Q_{19}=-0.41, Q_{20}=-0.66, Q_{21}=-0.11, Q_{22}=0.13$. Cartesian coordinates for the geometry of the minimum energy conical intersection can be found in supplementary information.

The conical intersection was calculated to be $0.54 \mathrm{eV}$ above the Franck-Condon point of the ${ }^{2} B_{1}$ state. The geometry is distorted away from the Franck-Condon point but remains planar. The distortion is similar to the quinoid-type vibration observed for benzene and phenyl derivatives. There is a lengthening of the ortho to meta $\mathrm{C}-\mathrm{C}$ bonds and contraction of the remaining $\mathrm{C}-\mathrm{C}$ bonds. The $\mathrm{C}-\mathrm{O}$ and the $\mathrm{O}-\mathrm{H}$ bonds both contract and the $\mathrm{C}-\mathrm{O}-\mathrm{H}$ angle increases. The derivative coupling and gradient difference vectors, showing the direction of the distortion by which the degeneracy is lifted, are illustrated in Figure 4.

A series of equal step points were generated from the Franck-Condon point, $Q_{0}$, to $Q_{\mathrm{CI}}$ and the energies at each point calculated using $\operatorname{CASSCF}(7,8)$. The fitted points along the vector to the intersection are shown in Figure 5. The fitting was up to second order only. As far as the authors are aware, this is the first reported observation of a conical intersection between these two states. The intersection is relatively high energy from the minima of the ${ }^{2} B_{1}$ and ${ }^{2} A_{2}$ states and is shifted far from $Q_{0}$, nevertheless a small amount of population transfer between states is possible.

\subsection{The Calculated spectra}

Figure 6 shows the calculated photoelectron spectrum (b and c) for transitions to the lowest ionised states with comparison to experiment (a). The band between 8.2 and $9 \mathrm{eV}$ is the transition to the ${ }^{2} B_{1}$ state and between 9.2 and 10 $\mathrm{eV}$ to the ${ }^{2} A_{2}$ state. The spectrum was calculated using the Quantics package, with the parameters describing the potential energy surfaces obtained from the 
fitting using VCHAM as described previously. For calculating the spectrum, a two state, seven mode model Hamiltonian was required including the $D_{0}\left({ }^{2} B_{1}\right)$ and $D_{1}\left({ }^{2} A_{2}\right)$ ionised states. Both spectral bands have been shifted to account for the zero point energy of the neutral molecule. The simulated spectrum shows good agreement with experiment. The electronic origins, $0_{0}^{0}$, for each band located at 8.33 and $9.31 \mathrm{eV}$ also show good agreement. For the ${ }^{2} B_{1}$ spectrum in Figure 6(b), a damping time of 30 fs was used whereas 14 fs was used for the ${ }^{2} A_{2}$ spectrum. The differences indicate that the modes ignored contribute less broadening to the ${ }^{2} B_{1}$ portion of the spectrum. The presence of the conical intersection also adds broadening to the ${ }^{2} A_{2}$ band.

The ${ }^{2} B_{1} \longleftarrow \tilde{X}^{1} A_{1}$ spectrum (left band in Figure 6 ) is well structured with progressions from several vibrational modes. Figure 7 (a) shows this band with an applied damping of 150 fs. This provides higher resolution to the spectrum and allows analysis of the peak progressions. In their assignment for this band, Debies and Rabalais attributed the structure to the symmetric ring deformation/C-O stretch and ring stretching modes with frequencies $530 \mathrm{~cm}^{-1}$ and $1608 \mathrm{~cm}^{-1}$, respectively [18]. In this work these correspond to modes $\nu_{1}$ and $\nu_{9}$, however only including these modes is not sufficient to fully assign, or indeed reproduce, the spectrum. The band origin $0_{0}^{0}$ is located at $8.33 \mathrm{eV}$ with the $\nu_{1}$ progression $1_{0}^{n}$ immediately following, replicating well both the spacing and relative peak height. The most intense peak in the spectrum forms from the $\mathrm{C}-\mathrm{C}$ ring stretch progression $9_{0}^{1}$. These modes on the own, however, are insufficient to explain the vibrational structure of the band and we therefore assign a peak to the $\mathrm{C}-\mathrm{O}$ stretch progression, $7_{0}^{1}$. Observed on both the $7_{0}^{1}$ and $9_{0}^{1}$ peak is a shoulder peak forming part of the long $1_{0}^{n}$ progression.

The ${ }^{2} A_{2} \longleftarrow \tilde{X}^{1} A_{1}$ spectrum (right band in Figure 6) is broader and lacks structure. In the original assignment, what little vibrational structure is present was assigned to another ring deformation/C-O stretch mode with a frequency of $814 \mathrm{~cm}^{-1}$. In our model this corresponds to mode $\nu_{2}$. From the cut along this mode and subsequent fitted parameters, it seems unlikely this mode is responsible for the observed structure and therefore we disagree in this assignment. Instead, the peak at $\sim 9.47 \mathrm{eV}$ is attributed to the $\mathrm{C}-\mathrm{O}$ stretch, $7_{0}^{1}$. The shoulder peak on the $0_{0}^{0}$ transition, which can be observed in the experimental spectrum, results from the $1_{0}^{1}$ progression. Figure $7(\mathrm{~b})$ shows there to be underlying vibrational structure, however both the $\nu_{1}$ and $\nu_{7}$ progressions rapidly decrease in intensity and are obscured in the broadness of the spectrum. The high resolution spectrum also show the presence of a peak corresponding to the $9_{0}^{1}$, which is not observable in the experimental or lower resolution simulated spectrum.

Dynamical information and the effect of the conical intersection were also investigated. Figure 8 shows the diabatic state populations of the ${ }^{2} B_{1}$ and ${ }^{2} A_{2}$ states during the simulation time of $200 \mathrm{fs}$, following ionisation to the ${ }^{2} A_{2}$ 
state. Within 10 fs significant population transfer occurs, indicating transfer of the wavepacket onto the lower state. Following this initial burst, the population of the ${ }^{2} B_{1}$ state increases gradually during the simulation, reaching a final population value of 0.3 at $200 \mathrm{fs}$. This supports the involvement of the conical intersection in the dynamics of phenol ionisation.

\section{Conclusions}

The study and rationalisation of molecular photodynamics can be explained through the topology of the potential energy surfaces. Consideration of these surfaces are especially critical where non-adiabaticity is present and vibronic coupling plays an important role. Accurately calculating the potential energy surfaces is vital for capturing the coupling correctly. Despite lacking dynamic electron correlation, the CASSCF method performs reasonably well in reproducing the surfaces. Even though the calculated ionisation energies are lower than those determined from experiments, the method correctly predicts the ordering of states and relative energetic difference.

A full multi-dimensional potential energy surface encompassing all the vibrational modes of a molecule is computationally demanding for all but the smallest systems. For phenol this means that explicit consideration of all 33 vibrational modes is unfeasible. Taking a smaller, select number of modes containing all the totally symmetric modes and the $b_{2}$ coupling modes, creates a more tractable model. The automatic fitting procedure is able to provide all the necessary parameters, but the linear optimisation used in the fitting does not guarantee the parameters obtained are optimal and a different set of parameters may be better. This is evident by the need to adjust several parameters to accurately reproduce the experimental spectrum.

In addition to the coupling between states, a conical intersection was found to exist. Whilst a cut along the lowest frequency totally symmetric mode showed the states to cross, the actual intersection was located along a vector comprising a large number of normal modes. Diabatic state populations during the simulation showed that ionisation into the ${ }^{2} A_{2}$ state leads to transfer and population of the lower ionised state.

The model described here, consisting of seven modes, is complete enough to reproduce the experimental photoelectron spectrum and thus describes the regions around the Franck-Condon point well. The analysis of the vibrational fine structure differs here to that proposed alongside the experimental data. An extra mode is needed to fully assign the first ionisation band. The little structure observed in the second ionisation band was suggested to correspond to the second lowest totally-symmetric vibrational mode. From the linear cou- 
pling parameters and cuts along the potential energy surface, an alternative assignment is suggested.

\section{Acknowledgement}

This paper is dedicated to Wolfgang Domcke on the occasion of his 70th birthday. The work was funded in part by the EPSRC through a DTP award at the University of Birmingham. 


\section{References}

[1] Z. Lan, W. Domcke, V. Vallet, A. L. Sobolewski, S. Mahapatra, Time-dependent quantum wave-packet description of the $\pi 1 \sigma^{*}$ photochemistry of phenol, J. Chem. Phys. 122 (2005) 224315.

[2] M. L. Hause, Y. Heidi Yoon, A. S. Case, F. F. Crim, Dynamics at conical intersections: The influence of $\mathrm{OH}$ stretching vibrations on the photodissociation of phenol, J. Chem. Phys. 128 (2008) 104307.

[3] R. N. Dixon, T. A. A. Oliver, M. N. R. Ashfold, Tunnelling under a conical intersection: Application to the product vibrational state distributions in the UV photodissociation of phenols, J. Chem. Phys. 134 (2011) 194303.

[4] X. Xu, K. R. Yang, D. G. Truhlar, Diabatic Molecular Orbitals, Potential Energies, and Potential Energy Surface Couplings by the 4-fold Way for Photodissociation of Phenol, J. Chem. Theory Comput. 9 (2013) 3612-3625.

[5] X. Xu, J. Zheng, K. R. Yang, D. G. Truhlar, Photodissociation Dynamics of Phenol: Multistate Trajectory Simulations including Tunneling, J. Am. Chem. Soc. 136 (2014) 16378-16386.

[6] X. Zhu, D. R. Yarkony, Fitting coupled potential energy surfaces for large systems: Method and construction of a 3-state representation for phenol photodissociation in the full 33 internal degrees of freedom using multireference configuration interaction determined data, J. Chem. Phys. 140 (2014) 024112.

[7] M. N. R. Ashfold, B. Cronin, A. L. Devine, R. N. Dixon, M. G. D. Nix, The role of $\pi \sigma^{*}$ excited states in the photodissociation of heteroaromatic molecules., Science 312 (2006) 1637-1640.

[8] G. M. Roberts, A. S. Chatterley, J. D. Young, V. G. Stavros, Direct Observation of Hydrogen Tunneling Dynamics in Photoexcited Phenol, J. Phys. Chem. Lett. 3 (2012) 348-352.

[9] A. L. Sobolewski, W. Domcke, Conical intersections induced by repulsive $1 \pi \sigma^{*}$ states in planar organic molecules: malonaldehyde, pyrrole and chlorobenzene as photochemical model systems, Chem. Phys. 259 (2000) 181-191.

[10] A. L. Sobolewski, W. Domcke, C. Dedonder-Lardeux, C. Jouvet, Excited-state hydrogen detachment and hydrogen transfer driven by repulsive $1 \pi \sigma^{*}$ states: A new paradigm for nonradiative decay in aromatic biomolecules, Phys. Chem. Chem. Phys. 4 (2002) 1093-1100.

[11] A. L. Sobolewski, W. Domcke, Ab initio investigations on the photophysics of indole, Chem. Phys. Lett. 315 (1999) 293-298.

[12] V. Vallet, Z. Lan, S. Mahapatra, A. L. Sobolewski, W. Domcke, Time-dependent quantum wave-packet description of the $1 \pi \sigma^{*}$ photochemistry of pyrrole, Faraday Discuss. 127 (2004) 283-293. 
[13] V. Vallet, Z. Lan, S. Mahapatra, A. L. Sobolewski, W. Domcke, Photochemistry of pyrrole: Time-dependent quantum wave-packet description of the dynamics at the $\pi 1 \sigma^{*}{ }_{-} \mathrm{S}_{0}$ conical intersections, J. Chem. Phys. 123 (2005) 144307.

[14] A. L. Sobolewski, W. Domcke, Photoinduced Electron and Proton Transfer in Phenol and its Clusters with Water and Ammonia, J. Phys. Chem. A 105 (2001) 9275-9283.

[15] C. P. Schick, S. D. Carpenter, P. M. Weber, Femtosecond Multiphoton Ionization Photoelectron Spectroscopy of the $\mathrm{S}_{2}$ State of Phenol, J. Phys. Chem. A 103 (1999) 10470-10476.

[16] R. A. Livingstone, J. O. F. Thompson, M. Iljina, R. J. Donaldson, B. J. Sussman, M. J. Paterson, D. Townsend, Time-resolved photoelectron imaging of excited state relaxation dynamics in phenol, catechol, resorcinol, and hydroquinone, J. Chem. Phys. 137 (2012) 184304.

[17] J. W. Riley, B. Wang, J. L. Woodhouse, M. Assmann, G. A. Worth, H. H. Fielding, Unravelling the Role of an Aqueous Environment on the Electronic Structure and Ionization of Phenol Using Photoelectron Spectroscopy, J. Phys. Chem. Lett. 9 (2018) 678-682.

[18] T. Debies, J. Rabalais, Photoelectron spectra of substituted benzenes: II. Seven valence electron substituents, J. Electron Spectros. Relat. Phenomena 1 (1972) $355-370$.

[19] J. P. Maier, D. W. Turner, Steric inhibition of resonance studied by molecular photoelectron spectroscopy. Part 3.Anilines, phenols and related compounds, J. Chem. Soc., Faraday Trans. 269 (1973) 521-531.

[20] T. Kobayashi, S. Nagakura, Photoelectron Spectra of Substituted Benzenes, Bull. Chem. Soc. Jpn. 47 (1974) 2563-2572.

[21] M. H. Palmer, W. Moyes, M. Speirs, J. A. Ridyard, The electronic structure of substituted benzenes; ab initio calculations and photoelectron spectra for phenol, the methyl- and fluoro-derivatives, and the dihydroxybenzenes, J. Mol. Struct. 52 (1979) 293-307.

[22] W. Domcke, G. Stock, Theory of ultrafast nonadiabatic excited-state processes and their spectroscopic detection in real time, Adv. Chem. Phys. 100 (1997) $1-169$.

[23] R. S. Minns, D. S. N. Parker, T. J. Penfold, G. A. Worth, H. H. Fielding, Competing ultrafast intersystem crossing and internal conversion in the "channel 3" region of benzene, PCCP 12 (2010) 15607-15615.

[24] G. Wu, S. P. Neville, O. Schalk, T. Sekikawa, M. N. R. Ashfold, G. A. Worth, A. Stolow, Excited state non-adiabatic dynamics of pyrrole: A time-resolved photoelectron spectroscopy and quantum dynamics study, J. Chem. Phys. 142 (2015) 074302-12. 
[25] M. Seel, W. Domcke, Model studies on femtosecond time-resolved ionization spectroscopy of excited-state vibrational dynamics and vibronic coupling, Chem. Phys. 151 (1990) 59-72.

[26] M. Seel, W. Domcke, Femtosecond time-resolved ionization spectroscopy of ultrafast internal-conversion dynamics in polyatomic molecules: Theory and computational studies, J. Chem. Phys. 95 (1991) 7806-7822.

[27] G. A. Worth, R. E. Carley, H. H. Fielding, Using photoelectron spectroscopy to unravel the excited-state dynamics of benzene, Chem. Phys. 338 (2007) 220227.

[28] H. Köppel, W. Domcke, L. S. Cederbaum, Multimode molecular dynamics beyond the Born-Oppenheimer approximation, Adv. Chem. Phys. 57 (1984) 59-246.

[29] G. A. Worth, H.-D. Meyer, H. Köppel, L. S. Cederbaum, I. Burghardt, Using the MCTDH wavepacket propagation method to describe multimode nonadiabatic dynamics, Int. Rev. Phys. Chem. 27 (2008) 569-606.

[30] G. Worth, A model Hamiltonian to simulate the complex photochemistry of benzene, J. Photochem. Photobiol. A Chem. 190 (2007) 190-199.

[31] T. J. Penfold, G. A. Worth, A model Hamiltonian to simulate the complex photochemistry of benzene II, J. Chem. Phys. 131 (2009) 064303.

[32] F. Wang, S. P. Neville, R. Wang, G. A. Worth, Quantum Dynamics Study of Photoexcited Aniline, J. Phys. Chem. A 117 (2013) 7298-7307.

[33] S. P. Neville, G. A. Worth, A reinterpretation of the electronic spectrum of pyrrole: A quantum dynamics study, J. Chem. Phys. 140 (2014) 034317.

[34] G. W. Richings, G. A. Worth, The time-resolved photoelectron spectrum of toluene using a perturbation theory approach, J. Chem. Phys. 141 (2014) 244115 .

[35] C. Cattarius, A. Markmann, G. A. Worth, The VCHAM program, see http://www.pci.uni-heidelberg.de/tc/usr/mctdh/ (2007).

[36] A. Worth, G. A. and Giri,K. and Richings, G. W. and Burghardt, I. and Beck,M. H. and Jäckle, H.-D. Meyer., The Quantics Package (2015).

[37] M. J. Frisch, G. W. Trucks, H. B. Schlegel, G. E. Scuseria, M. A. Robb, J. R. Cheeseman, G. Scalmani, V. Barone, G. A. Petersson, H. Nakatsuji, X. Li, M. Caricato, A. V. Marenich, J. Bloino, B. G. Janesko, R. Gomperts, B. Mennucci, H. P. Hratchian, J. V. Ortiz, A. F. Izmaylov, J. L. Sonnenberg, D. Williams-Young, F. Ding, F. Lipparini, F. Egidi, J. Goings, B. Peng, A. Petrone, T. Henderson, D. Ranasinghe, V. G. Zakrzewski, J. Gao, N. Rega, G. Zheng, W. Liang, M. Hada, M. Ehara, K. Toyota, R. Fukuda, J. Hasegawa, M. Ishida, T. Nakajima, Y. Honda, O. Kitao, H. Nakai, T. Vreven, K. Throssell, J. A. Montgomery Jr., J. E. Peralta, F. Ogliaro, M. J. Bearpark, J. J. Heyd, E. N. Brothers, K. N. Kudin, V. N. Staroverov, T. A. Keith, R. Kobayashi, 
J. Normand, K. Raghavachari, A. P. Rendell, J. C. Burant, S. S. Iyengar, J. Tomasi, M. Cossi, J. M. Millam, M. Klene, C. Adamo, R. Cammi, J. W. Ochterski, R. L. Martin, K. Morokuma, O. Farkas, J. B. Foresman, D. J. Fox, Gaussian 09 (2009).

[38] M. Beck, The multiconfiguration time-dependent Hartree (MCTDH) method: a highly efficient algorithm for propagating wavepackets, Phys. Rep. 324 (2000) $1-105$.

[39] R. J. Lipert, S. D. Colson, Accurate ionization potentials of phenol and phenol $\left(\mathrm{H}_{2} \mathrm{O}\right)$ from the electric field dependence of the pumpprobe photoionization threshold, J. Chem. Phys. 92 (1990) 3240-3241.

[40] J. Evans, The vibrational spectra of phenol and phenol-OD, Spectrochim. Acta 16 (1960) 1382-1392.

[41] S. Schumm, M. Gerhards, W. Roth, H. Gier, K. Kleinermanns, A CASSCF study of the $\mathrm{S}_{0}$ and $\mathrm{S}_{1}$ states of phenol, Chem. Phys. Lett. 263 (1996) 126-132.

[42] P. J. O'Malley, Electronic structure calculated anharmonic vibrational frequencies for phenol, J. Mol. Struct. (Theochem) 755 (2005) 147-150.

[43] H. Lampert, W. Mikenda, A. Karpfen, Molecular geometries and vibrational spectra of phenol, benzaldehyde, and salicylaldehyde: Experimental versus quantum chemical data, J. Phys. Chem. A 101 (1997) 2254-2263.

[44] D. Michalska, D. C. Bieńko, A. J. Abkowicz-Bieńko, Z. Latajka, Density functional, Hartree-Fock, and MP2 studies on the vibrational spectrum of phenol, J. Phys. Chem. 100 (1996) 17786-17790.

[45] H. Bist, J. Brand, D. Williams, The vibrational spectrum and torsion of phenol, J. Mol. Spectrosc. 24 (1967) 402-412.

[46] K. R. Yang, X. Xu, J. Zheng, D. G. Truhlar, Full-dimensional potentials and state couplings and multidimensional tunneling calculations for the photodissociation of phenol, Chem. Sci. 5 (2014) 4661-4680. 
Table 1

Computational details for the quantum dynamic simulations. The DVR type HO corresponds to Harmonic oscillator DVR, $N_{i}$ are the number of primitive DVR functions used to describe each mode and $n_{i}$ are the number of single particle functions used for the wavepacket on each state

\begin{tabular}{lcccc}
\hline & Mode & DVR Type & $N_{i}$ & $n_{1}, n_{2}$ \\
\hline Excitation to ${ }^{2} B_{1}$ & $Q_{1}$ & HO & 31 & 6,7 \\
$Q_{6}$ & HO & 31 & 6,7 \\
$Q_{7}$ & HO & 31 & 6,7 \\
$Q_{9}$ & HO & 31 & 6,7 \\
$Q_{12}$ & HO & 31 & 6,7 \\
& $Q_{13}$ & HO & 31 & 6,7 \\
Excitation to ${ }^{2} A_{2}$ & $Q_{14}$ & HO & 31 & 6,7 \\
& $Q_{1}$ & HO & 31 & 6,7 \\
& $Q_{6}$ & HO & 31 & 6,7 \\
$Q_{7}$ & HO & 31 & 6,7 \\
$Q_{9}$ & HO & 31 & 6,7 \\
& $Q_{12}$ & HO & 31 & 6,7 \\
& $Q_{13}$ & HO & 31 & 10,7 \\
$Q_{14}$ & HO & 31 & 10,7 \\
\hline
\end{tabular}

Table 2

Vertical ionisation energies of phenol, calculated from the equilibrium geometry. The $\operatorname{CAS}(7,8)$ calculations used a $6-31+\mathrm{G}^{*}$ basis. All values in $\mathrm{eV}$.

\begin{tabular}{lrr}
\hline State & $\mathrm{CAS}(7,8)$ & Experimental \\
\hline${ }^{2} B_{1}$ & 7.986 & $8.508[39]$ \\
${ }^{2} A_{2}$ & 8.670 & $9.280[21]$ \\
\hline
\end{tabular}


Table 3

Theoretically calculated frequencies of vibrational modes, in $\mathrm{cm}^{-1}$ and electron volts, for $\tilde{X}^{1} A_{1}$ state of phenol calculated at the MP2/aug-cc-pVDZ level of theory and ordered by their $\mathrm{C}_{2 v}$ symmetries.

\begin{tabular}{|c|c|c|c|c|c|}
\hline \multirow[b]{2}{*}{ Mode } & \multicolumn{2}{|c|}{ Frequency } & \multicolumn{2}{|c|}{ Symmetry } & \multirow[t]{2}{*}{ Description } \\
\hline & $/ \mathrm{cm}^{-1}$ & $/ \mathrm{eV}$ & $\left(C_{s}\right)$ & $\left(C_{2 v}\right)[40]$ & \\
\hline 1 & 520 & 0.0645 & $A^{\prime}$ & $a_{1}$ & Ring deformation $+\mathrm{C}-\mathrm{O}$ stretch \\
\hline 2 & 812 & 0.1007 & $A^{\prime}$ & $a_{1}$ & Ring deformation $+\mathrm{C}-\mathrm{O}$ stretch \\
\hline 3 & 998 & 0.1238 & $A^{\prime}$ & $a_{1}$ & Breathing mode \\
\hline 4 & 1035 & 0.1284 & $A^{\prime}$ & $a_{1}$ & $\mathrm{C}-\mathrm{H}$ bend in plane \\
\hline 5 & 1176 & 0.1458 & $A^{\prime}$ & $a_{1}$ & C-H bend \\
\hline 6 & 1194 & 0.1480 & $A^{\prime}$ & $a_{1}$ & $\mathrm{OH}$ bend \\
\hline 7 & 1267 & 0.1571 & $A^{\prime}$ & $a_{1}$ & $\mathrm{C}-\mathrm{O}$ stretch $+\mathrm{C}-\mathrm{C}$ stretch \\
\hline 8 & 1501 & 0.1861 & $A^{\prime}$ & $a_{1}$ & $\mathrm{C}-\mathrm{C}$ ring stretch \\
\hline 9 & 1646 & 0.2041 & $A^{\prime}$ & $a_{1}$ & $\mathrm{C}-\mathrm{C}$ ring stretch \\
\hline 10 & 3192 & 0.3957 & $A^{\prime}$ & $a_{1}$ & C-H stretch \\
\hline 11 & 3236 & 0.4013 & $A^{\prime}$ & $a_{1}$ & C-H stretch \\
\hline 12 & 3806 & 0.4719 & $A^{\prime}$ & $a_{1}$ & O-H stretch \\
\hline 13 & 397 & 0.0492 & $A^{\prime}$ & $b_{2}$ & In plane $\mathrm{C}-\mathrm{O}-\mathrm{H}$ bend \\
\hline 14 & 612 & 0.0758 & $A^{\prime}$ & $b_{2}$ & Ring deformation \\
\hline 15 & 1083 & 0.1343 & $A^{\prime}$ & $b_{2}$ & $\mathrm{C}-\mathrm{H}$ bend in plane \\
\hline 16 & 1161 & 0.1440 & $A^{\prime}$ & $b_{2}$ & $\mathrm{C}-\mathrm{H}$ bend in plane \\
\hline 17 & 1332 & 0.1652 & $A^{\prime}$ & $b_{2}$ & $\mathrm{C}-\mathrm{H}$ bend \\
\hline 18 & 1471 & 0.1823 & $A^{\prime}$ & $b_{2}$ & C-C ring stretch \\
\hline 19 & 1482 & 0.1837 & $A^{\prime}$ & $b_{2}$ & $\mathrm{C}-\mathrm{C}$ ring stretch \\
\hline 20 & 1635 & 0.2027 & $A^{\prime}$ & $b_{2}$ & C-C ring stretch \\
\hline 21 & 3207 & 0.3977 & $A^{\prime}$ & $b_{2}$ & C-H stretch \\
\hline 22 & 3217 & 0.3988 & $A^{\prime}$ & $b_{2}$ & C-H stretch \\
\hline 23 & 3230 & 0.4004 & $A^{\prime}$ & $b_{2}$ & C-H stretch \\
\hline 24 & 406 & 0.0504 & $A^{\prime \prime}$ & $a_{2}$ & C-C twist \\
\hline 25 & 817 & 0.1013 & $A^{\prime \prime}$ & $a_{2}$ & $\mathrm{C}-\mathrm{H}$ bend \\
\hline 26 & 910 & 0.1129 & $A^{\prime \prime}$ & $a_{2}$ & $\mathrm{C}-\mathrm{H}$ bend out of plane \\
\hline 27 & 223 & 0.0277 & $A^{\prime \prime}$ & $b_{1}$ & Out of plane $\mathrm{C}-\mathrm{O}$ bend + ring torsion \\
\hline 28 & 332 & 0.0412 & $A^{\prime \prime}$ & $b_{1}$ & Out of plane $\mathrm{O}-\mathrm{H}$ bend \\
\hline 29 & 496 & 0.0615 & $A^{\prime \prime}$ & $b_{1}$ & Out of plane $\mathrm{C}-\mathrm{O}$ bend + ring torsion \\
\hline 30 & 613 & 0.0760 & $A^{\prime \prime}$ & $b_{1}$ & Ring deformation \\
\hline 31 & 742 & 0.0920 & $A^{\prime \prime}$ & $b_{1}$ & $\mathrm{C}-\mathrm{H}$ bend out of plane \\
\hline 32 & 864 & 0.1071 & $A^{\prime \prime}$ & $b_{1}{ }^{19}$ & $\mathrm{C}-\mathrm{H}$ bend out of plane \\
\hline 33 & 926 & 0.1148 & $A^{\prime \prime}$ & $b_{1}$ & $\mathrm{C}-\mathrm{H}$ bend out of plane \\
\hline
\end{tabular}


Table 4

On-diagonal linear coupling constants, $\kappa_{\alpha}$ and off-diagonal linear coupling constants, $\lambda_{\alpha}$, for the select normal modes of phenol, as determined from fitting the vibronic coupling model Hamiltonian to adiabatic surfaces calculated at the $\operatorname{CASSCF}(7,8) / 6$ -

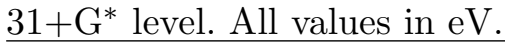

\begin{tabular}{lccc}
\hline & & $\kappa_{\alpha}$ & $\lambda_{\alpha}$ \\
\hline$\nu_{1}$ & ${ }^{2} B_{1}$ & ${ }^{2} A_{2}$ & - \\
$\nu_{2}$ & -0.118 & 0.051 & - \\
$\nu_{3}$ & -0.070 & -0.074 & - \\
$\nu_{4}$ & 0.001 & -0.011 & - \\
$\nu_{5}$ & 0.012 & 0.017 & - \\
$\nu_{6}$ & -0.089 & 0.044 & - \\
$\nu_{7}$ & -0.126 & -0.122 & - \\
$\nu_{8}$ & -0.129 & -0.138 & - \\
$\nu_{9}$ & -0.045 & -0.083 & - \\
$\nu_{10}$ & 0.111 & -0.070 & - \\
$\nu_{11}$ & -0.003 & -0.012 & - \\
$\nu_{12}$ & -0.008 & -0.034 & - \\
$\nu_{13}$ & -0.035 & -0.159 & -0.0002 \\
$\nu_{14}$ & - & - & 0.0011 \\
\hline
\end{tabular}

Table 5

Values of selected coupling parameters adjusted from those obtained from the fitting procedure in order to reproduce the experimental spectra. All values in $\mathrm{eV}$.

$\lambda_{13}$

$-0.100$

$\lambda_{14}$

0.080

$\kappa_{9}^{(1)}$

$-0.200$

$\gamma_{1-6}^{(1)}$

$-0.010$

$\gamma_{1-6}^{(2)}$

0.005

$\gamma_{1-9}^{(2)}$

$-0.010$

$\gamma_{6-7}^{(2)}$

$-0.020$

$\gamma_{6-9}^{(2)}$

$-0.020$ 


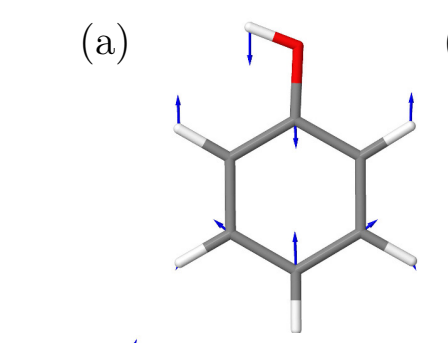

(b)

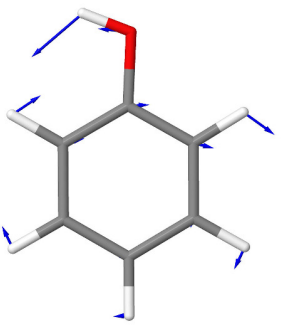

(c)
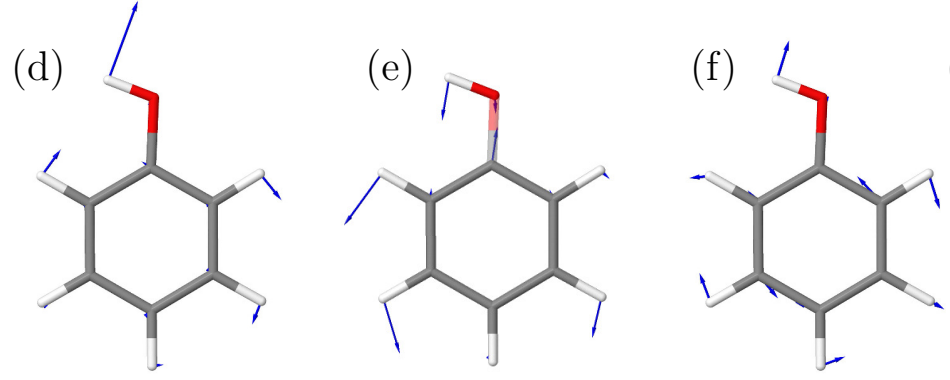

(g)

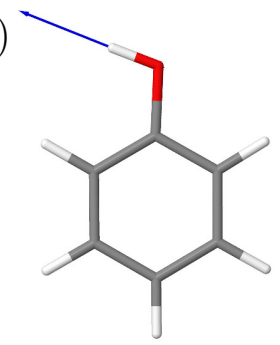

Fig. 1. The normal modes of phenol important in describing its photoelectron spectrum: (a) $\nu_{1}$ ring deformation and C-O stretch, (b) $\nu_{13}$ in plane C-O-H bend mode, (c) $\nu_{14}$ ring deformation mode, (d) $\nu_{6} \mathrm{O}-\mathrm{H}$ bend mode, (e) $\nu_{7} \mathrm{C}-\mathrm{O}$ stretch and ring distortion mode, (f) $\nu_{9} \mathrm{C}-\mathrm{C}$ ring stretch mode and (g) $\nu_{12} \mathrm{O}-\mathrm{H}$ stretch. 

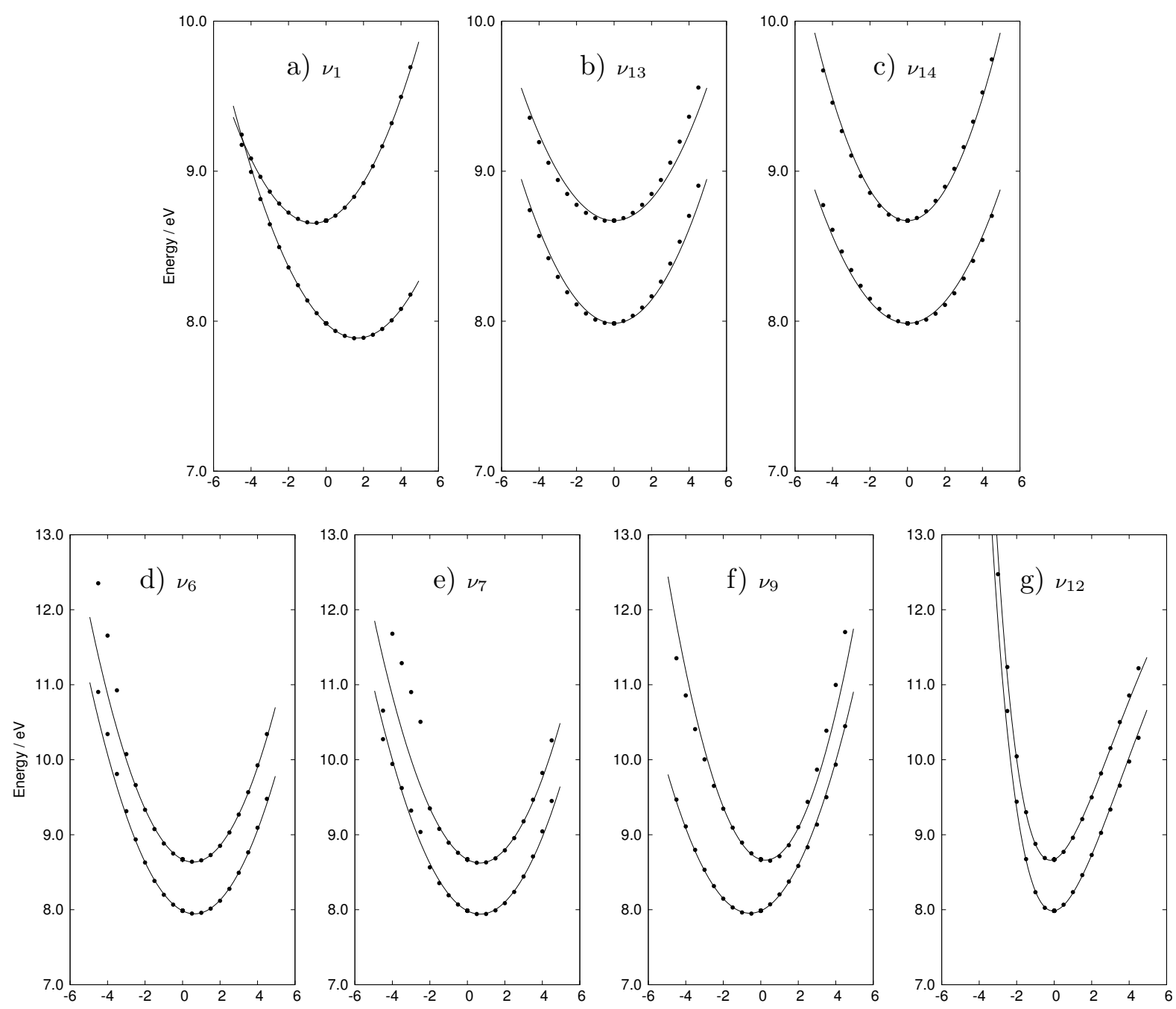

Fig. 2. Cuts through the adiabatic potential energy surfaces along select normal modes for the ionised states, which in order of energy at $Q_{0}$ are ${ }^{2} B_{1}$ and ${ }^{2} A_{2}$. The points are obtained from $a b$ initio calculations at the $\operatorname{CASSCF}(7,8) / 6-31+\mathrm{G}^{*}$ level. The normal modes included are: (a) $\nu_{1}$ ring deformation and C-O stretch, (b) $\nu_{13}$ in plane $\mathrm{C}-\mathrm{O}-\mathrm{H}$ bend mode, (c) $\nu_{14}$ ring deformation mode, (d) $\nu_{6} \mathrm{O}-\mathrm{H}$ bend mode, (e) $\nu_{7} \mathrm{C}$-O stretch mode, (f) $\nu_{9} \mathrm{C}-\mathrm{C}$ ring stretch mode and (g) $\nu_{12} \mathrm{O}-\mathrm{H}$ stretch. 


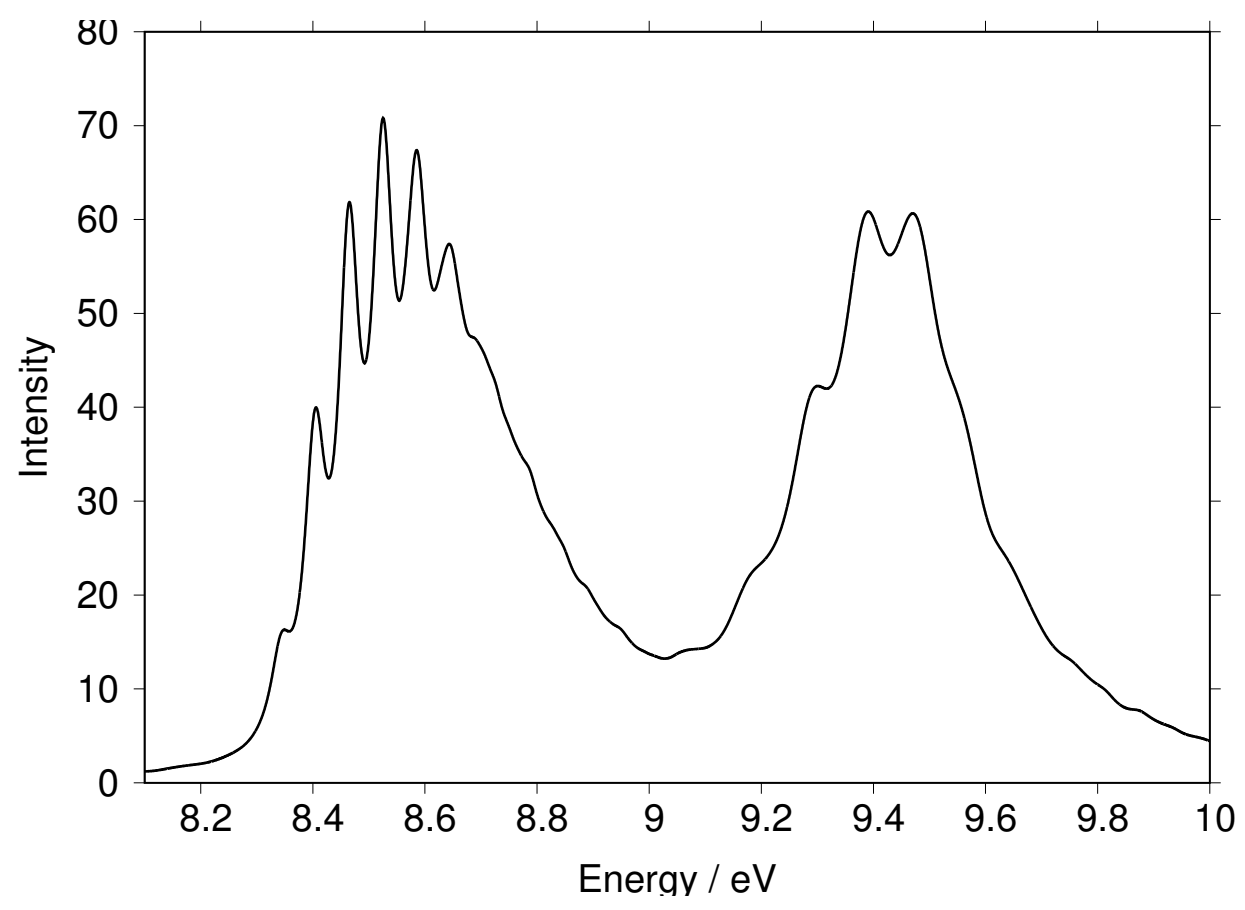

Fig. 3. The ${ }^{2} B_{1} \longleftarrow \tilde{X}^{1} A_{1}$ and ${ }^{2} A_{2} \longleftarrow \tilde{X}^{1} A_{1}$ photoelectron spectrum calculated using parameters obtained from the fitting procedure without any adjustments. The spectrum was calculated using the Quantics program and a seven mode model with a damping time of $30 \mathrm{fs}$ for the first band and $14 \mathrm{fs}$ for the second band.
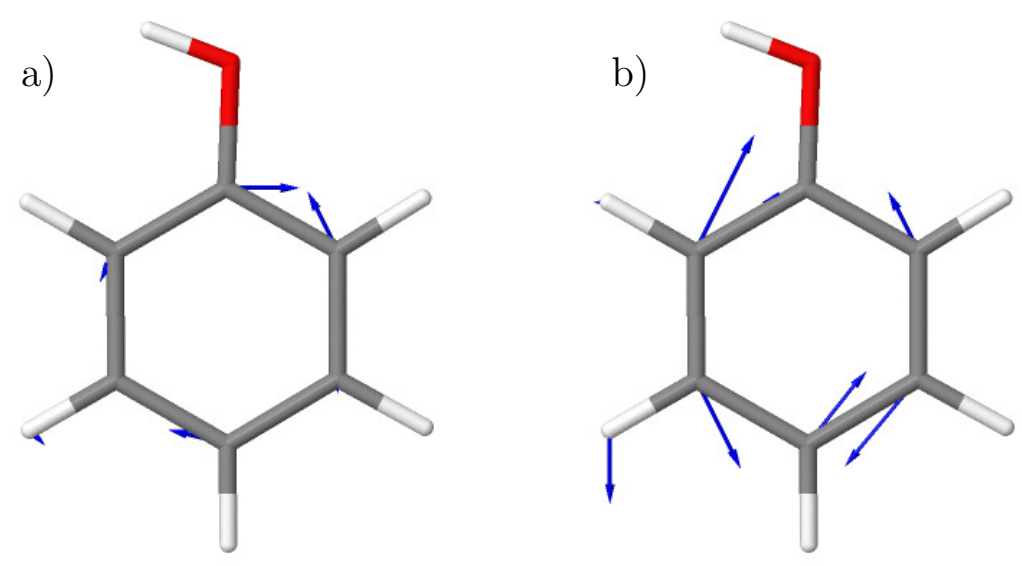

Fig. 4. The vectors leading to the conical intersection: (a) the derivative coupling vector and (b) the gradient difference vector. 


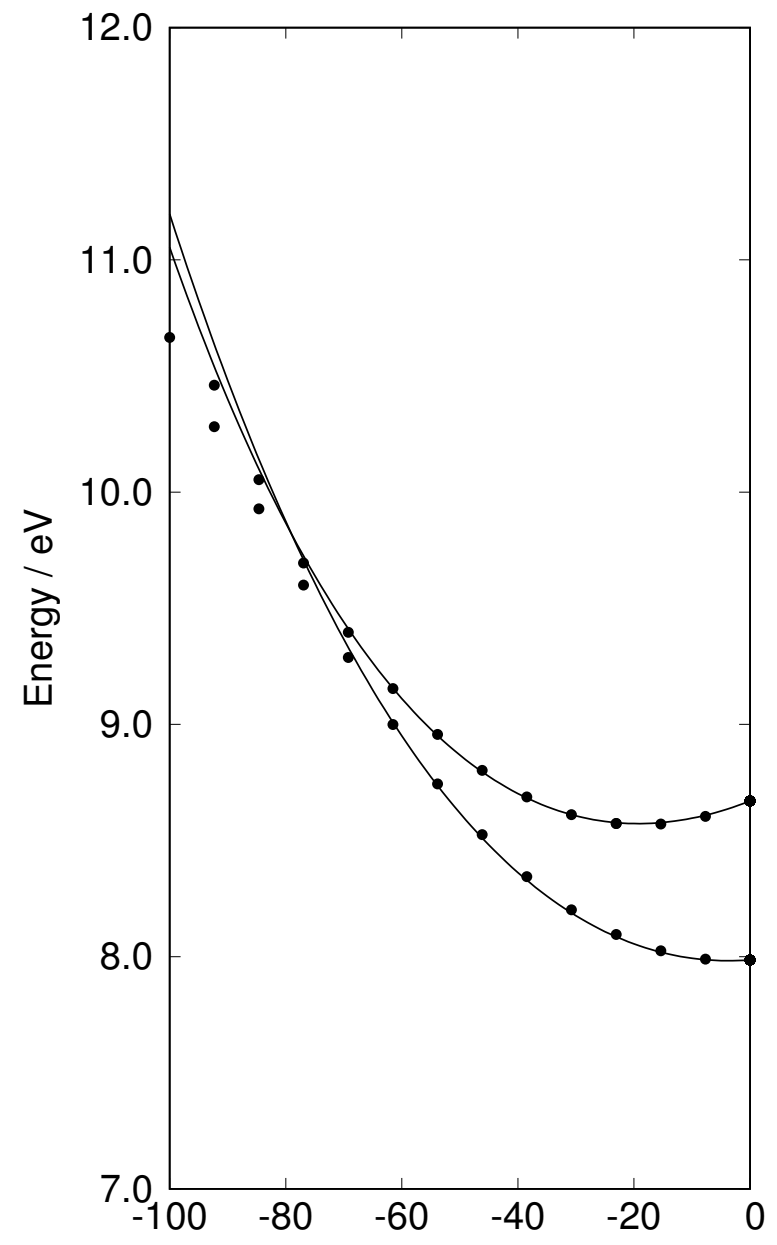

Fig. 5. Cut along the vector leading to the ${ }^{2} B_{1} /{ }^{2} A_{2}$ conical intersection. The points are obtained from $a b$ initio calculations at the $\operatorname{CASSCF}(7,8) / 6-31+\mathrm{G}^{*}$ level. 

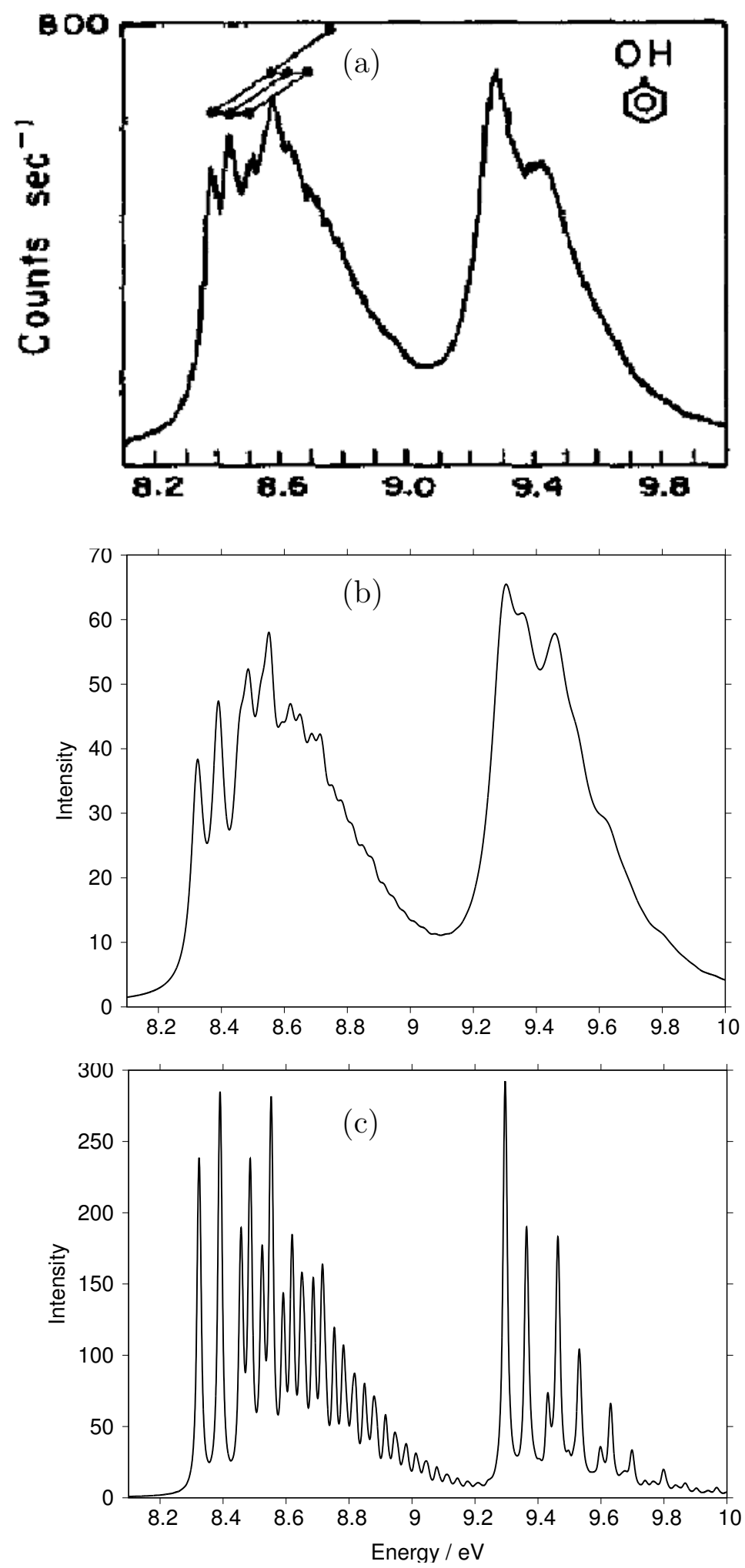

Fig. 6. The ${ }^{2} B_{1} \longleftarrow \tilde{X}^{1} A_{1}$ and ${ }^{2} A_{2} \longleftarrow \tilde{X}^{1} A_{1}$ photoelectron spectrum. (a) the experimental spectrum as measured by Debais and Rabalais and reproduced with permission [18]. (b) the calculated spectrum using the Quantics program and a seven mode model with a damping time of 30 fs for the first band and $14 \mathrm{fs}$ for the second band. (c) the calculated spectrum with a damping of $100 \mathrm{fs}$ to highlight the fine structure present. 

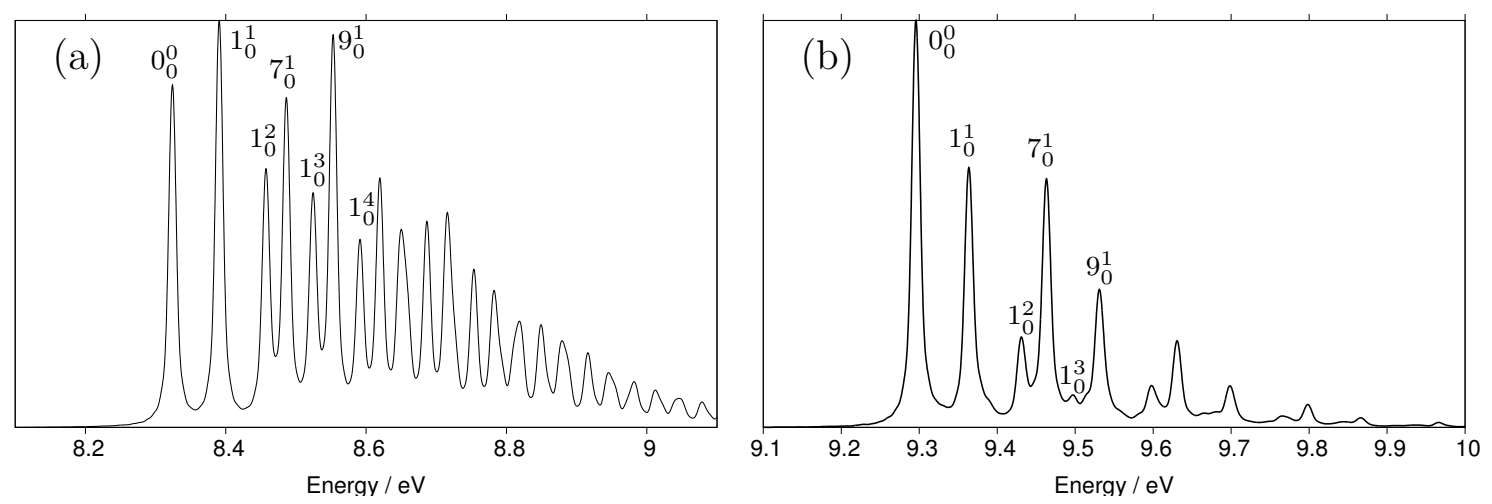

Fig. 7. High resolution photoelectron spectra for (a) ${ }^{2} B_{1} \longleftarrow \tilde{X}^{1} A_{1}$ and (b) ${ }^{2} A_{2} \longleftarrow \tilde{X}^{1} A_{1}$ transition. Assignments of the structure corresponding to the ground state vibrational modes are also shown. The higher resolution is achieved by setting the damping time to $150 \mathrm{fs}$.

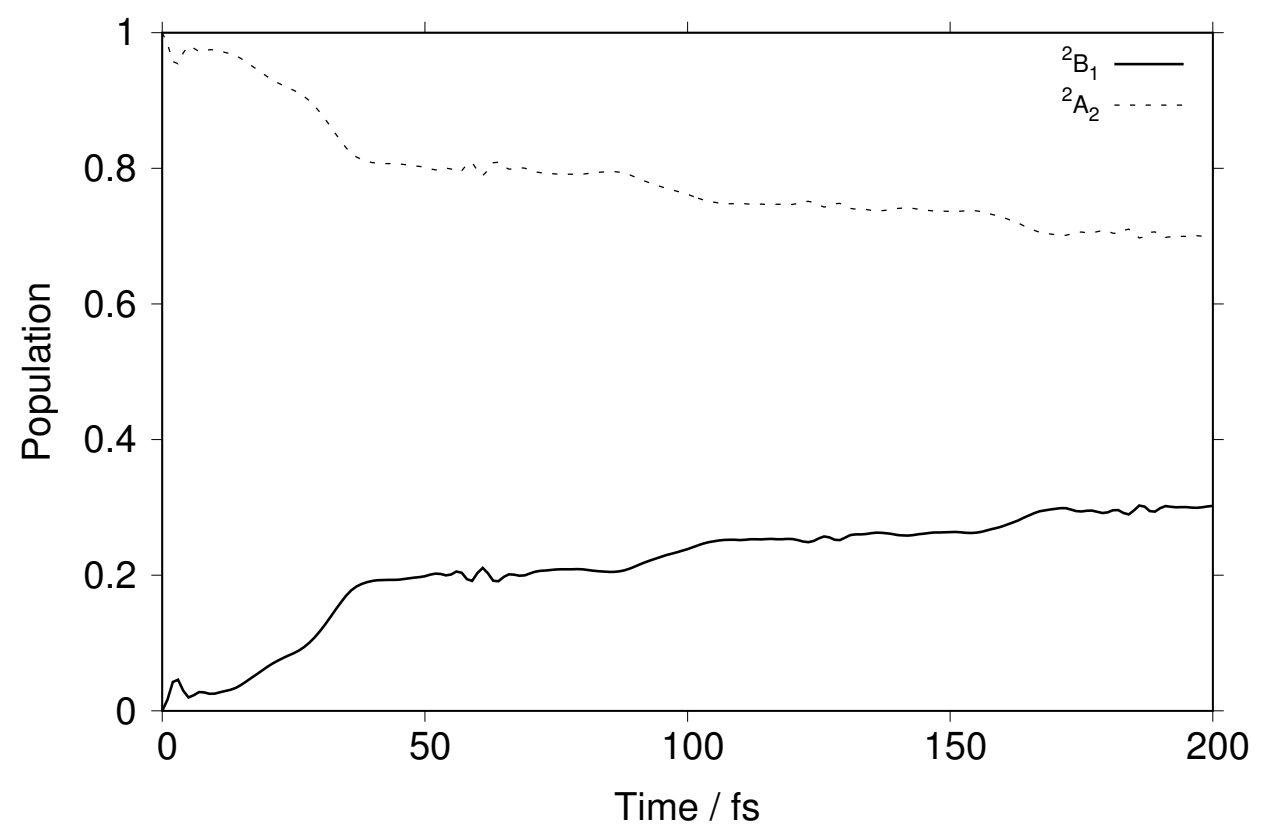

Fig. 8. State populations and transfer between the ${ }^{2} B_{1}$ and ${ }^{2} A_{2}$ states following a vertical ionisation into the ${ }^{2} A_{2}$ state. At the end of the simulation the population of ${ }^{2} A_{2}$ and ${ }^{2} B_{1}$ is approximately 0.7 and 0.3 , respectively. 